EGU2020-11982

EGU General Assembly 2020

(c) Author(s) 2020. This work is distributed under

the Creative Commons Attribution 4.0 License.

\title{
Advanced Bridge Monitoring Strategies by Polarimetric GB-SAR
}

\author{
Lilong Zou' ${ }^{1}$, Motoyuki Sato ${ }^{2}$, Fabio Tosti ${ }^{1}$, and Amir M. Alani ${ }^{1}$ \\ ${ }^{1}$ School of Computing and Engineering, University of West London (UWL), London, United Kingdom of Great Britain and \\ Northern Ireland (Lilong.Zou@uwl.ac.uk; Fabio.Tosti@uwl.ac.uk; Amir.Alani@uwl.ac.uk) \\ ${ }^{2}$ Center for Northeast Asian Studies, Tohoku University, Sendai, Japan (Motoyuki.Sato.B3@tohoku.ac.jp)
}

Use of advanced health monitoring strategies for bridges is essential to provide a comprehensive condition assessment of these structures and ensure their structural integrity. To this purpose, new technologies have been applied in recent years for both static and dynamic assessments offering unprecedented opportunities within the context of risk management and structural analysis. Among these, areal deformation measurement techniques from ground-based synthetic aperture radar (GB-SAR) sensors were successfully applied for continuous monitoring of dynamic and static displacements of bridges [1] [2]. However, a main limitation for the ground-based microwave interferometry is that, as a linear measurement technique, it is difficult to pinpoint the damage location and obtain accurate displacement time-series for bridges [3]. Moreover, it is known that vertical displacements are usually more relevant than horizontal displacements in the dynamic monitoring of bridges, and the GB-SAR interferometry can only provide the line-of-sight (LOS) displacement of the monitored bridge [4].

In this research, we focus on remote monitoring of the dynamic displacement responses of bridges with a polarimetric GB-SAR system. To this purpose, various strategies were used to overcome the existing limitations of this technique. Results from the monitoring of a long-span metallic railway bridge and a reinforced concrete Shinkansen bridge are discussed.

The aim of this research is to provide more comprehensive and accurate information for bridge health monitoring using a polarimetric sensor. To this extent, a polarimetric analysis was performed to identify the reflection from the side surface of the bridges. In addition, the information about the polarisation orientation angle and the local incidence angle were processed under the acquisition geometry to calculate the radar look angle. Therefore, the bridge deformation fields in the vertical direction were easily converted using the slant range distances and the corresponding maximum transient vertical deformation was transformed through the LOS deformation while a train passing the bridge.

\section{References}

[1] Monserrat, O. et al., 2014. A review of ground-based SAR Interferometry for deformation measurement. ISPRS Journal of Photogrammetry and Remote Sensing, pp. 40-48. 
[2] Pieraccini, M. et al., 2006. Dynamic monitoring of bridges using a high-speed coherent radar. IEEE Transaction Geoscience and Remote Sensing, pp. 3284-3288.

[3] Sato M., Zou L., Nico G., 2017. Monitoring of Infrastructure by GB-SAR. IEICE technical report, pp. 11-16.

[4] Sato M., Zou L., Nico G., Kikuta K., 2019. Displacement and Vibration Monitoring by GB-SAR. IEICE Transactions on Communications, pp.844-852. 